\title{
A Vision of 6G Wireless Systems: Applications, Trends, Technologies, and Open Research Problems
}

Walid Saad, Mehdi Bennis, and Mingzhe Chen

\section{ABSTRACT}

The ongoing deployment of 5G cellular systems is continuously exposing the inherent limitations of this system, compared to its original premise as an enabler for Internet of Everything applications. These 5G drawbacks are spurring worldwide activities focused on defining the next-generation 6G wireless system that can truly integrate far-reaching applications ranging from autonomous systems to extended reality. Despite recent 6G initiatives (one example is the 6Genesis project in Finland), the fundamental architectural and performance components of $6 \mathrm{G}$ remain largely undefined. In this article, we present a holistic, forward-looking vision that defines the tenets of a $6 \mathrm{G}$ system. We opine that 6G will not be a mere exploration of more spectrum at high-frequency bands, but it will rather be a convergence of upcoming technological trends driven by exciting, underlying services. In this regard, we first identify the primary drivers of $6 \mathrm{G}$ systems, in terms of applications and accompanying technological trends. Then, we propose a new set of service classes and expose their target 6G performance requirements. We then identify the enabling technologies for the introduced 6G services and outline a comprehensive research agenda that leverages those technologies. We conclude by providing concrete recommendations for the roadmap toward 6G. Ultimately, the intent of this article is to serve as a basis for stimulating more out-of-the-box research around 6G.

\section{INTRODUCTION}

To date, the wireless network evolution was primarily driven by a need for higher rates, which mandated a continuous 1000x increase in network capacity. While this demand for wireless capacity will continue to grow, the emergence of the Internet of Everything ( $\mathrm{loE}$ ) system, connecting millions of people and billions of machines, is yielding a radical paradigm shift from the rate-centric enhanced mobile broadband (eMBB) services of yesteryears toward ultra-reliable, low latency communications (URLLC).

Although the fifth generation (5G) cellular system was marketed as the key loE enabler, through concerted 5G standardization efforts that led to the first $5 \mathrm{G}$ new radio (5G NR) milestone and subsequent 3GPP releases, the initial premise of $5 \mathrm{G}-$ as a true carrier of loE services - is yet to be realized. One can argue that the evolutionary part of 5G (i.e., supporting rate-hungry eMBB services) has gained significant momentum. However, the promised revolutionary outlook of 5G, a system operating almost exclusively at high-frequency millimeter wave (mmWave) frequencies and enabling heterogeneous IoE services, has thus far remained a mirage. Although the $5 \mathrm{G}$ systems that are currently being marketed will readily support basic IoE and URLLC services (e.g., factory automation), it is debatable whether they can deliver tomorrow's smart city loE applications. Moreover, although $5 \mathrm{G}$ will eventually support fixed-access at $\mathrm{mmWave}$ frequencies, it is more likely that early $5 \mathrm{G}$ roll-outs will still use sub-6 $\mathrm{GHz}$ for supporting mobility.

Meanwhile, an unprecedented proliferation of new loE services is ongoing. Examples range from extended reality (XR) services (encompassing augmented, mixed, and virtual reality (AR/ MR/VR)) to telemedicine, haptics, flying vehicles, brain-computer interfaces, and connected autonomous systems. These applications will disrupt the original 5G goal of supporting short-packet, sensing-based URLLC services. To successfully operate IoE services such as XR and connected autonomous systems, a wireless system must simultaneously deliver high reliability, low latency, and high data rates, for heterogeneous devices, across uplink and downlink. Emerging loE services will also require an end-to-end co-design of communication, control, and computing functionalities, which to date has been largely overlooked. To cater to this new breed of services, unique challenges must be addressed ranging from characterizing the fundamental rate-reliability-latency tradeoffs governing their performance to exploiting frequencies beyond sub- $6 \mathrm{GHz}$ and transforming wireless systems into a self-sustaining, intelligent network fabric which flexibly provisions and orchestrates communication-computing-control-localization-sensing resources tailored to the requisite loE scenario.

To overcome these challenges, a disruptive sixth generation (6G) wireless system, whose design is inherently tailored to the performance requirements of IoE applications and their accompanying technological trends, is needed. The drivers of $6 \mathrm{G}$ will be a confluence of past trends (e.g., densification, higher rates, and massive antennas) and of emerging trends that include new services and the recent revolution in 


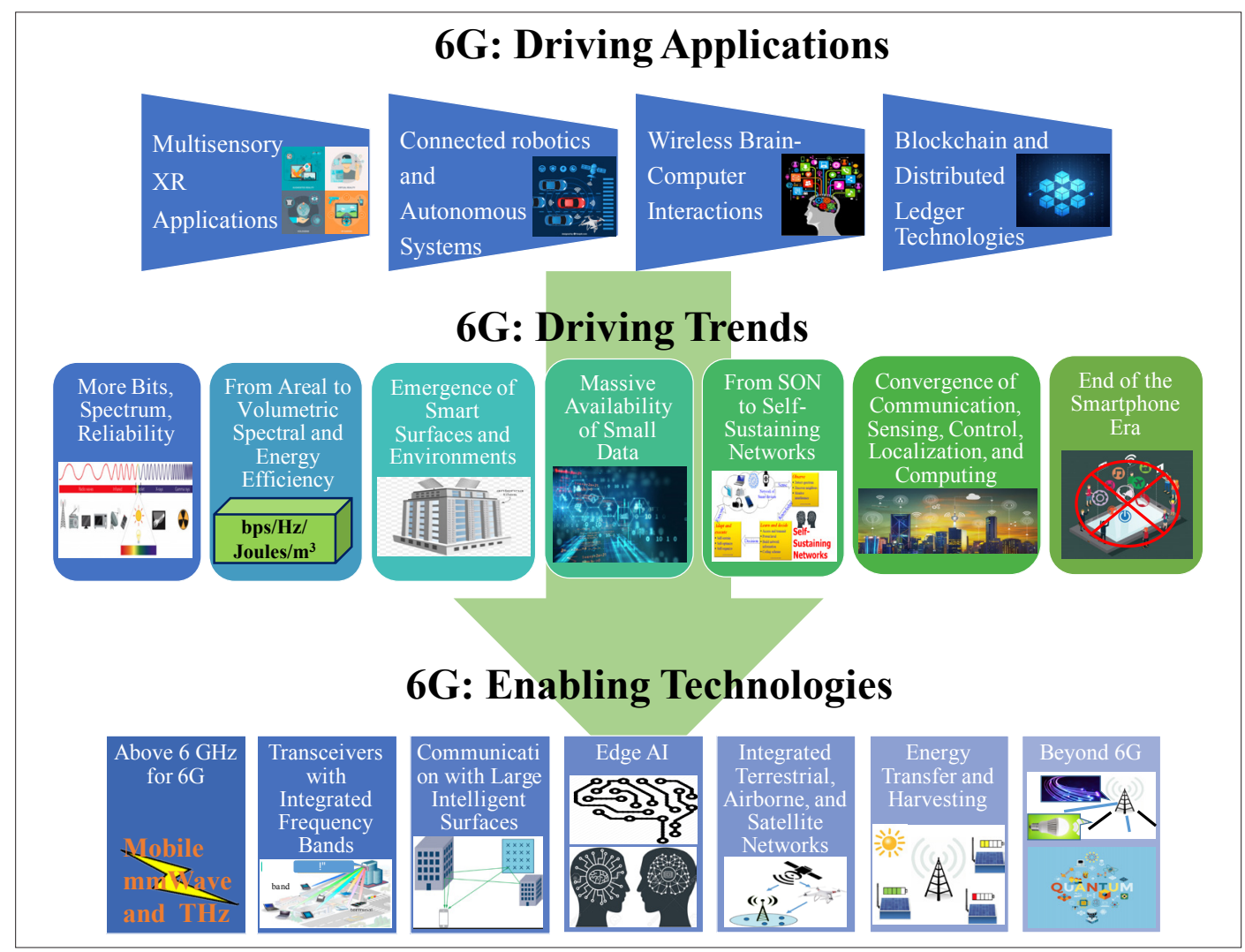

FIGURE 1.6G vision: applications, trends, and technologies.

wireless devices (e.g., smart wearables, implants, $\mathrm{XR}$ devices, and so on), artificial intelligence (AI) [1], computing, and sensing.

The main contribution of this article is a bold, forward-looking vision of 6G systems (Fig. 1) that identifies the applications, trends, and disruptive technologies, that will drive the $6 \mathrm{G}$ revolution. This vision will then delineate new $6 \mathrm{G}$ services and provide a concrete research roadmap and recommendations to facilitate the leap from current $5 \mathrm{G}$ systems toward $6 \mathrm{G}$.

\section{G DRIVING APPLICATIONS, MetricS, ANd NeW SeRVICE CLASSES}

Every new cellular generation is driven by innovative applications. $6 \mathrm{G}$ is no exception: It will be borne out of an unparalleled emergence of exciting new applications and technological trends that will shape its performance targets while radically redefining standard $5 \mathrm{G}$ services. Next, we introduce the main applications that motivate $6 \mathrm{G}$ deployment, and then discuss ensuing technological trends, target performance metrics, and new service requirements.

\section{Diling AppLICATIONS Behlind 6G And Thelr ReQuiREMENTS}

While traditional applications, such as live multimedia streaming, will remain central to $6 \mathrm{G}$, the key determinants of the system performance will be four new application domains.

Multisensory XR Applications: XR will yield many killer applications for 6G across the AR/ MR/VR spectrum. Upcoming 5G systems still fall short of providing a full immersive XR experience capturing all sensory inputs due to their inability to deliver very low latencies for data- rate intensive $X R$ applications. A truly immersive $A R / M R / V R$ experience requires a joint design integrating not only engineering (wireless, computing, storage) requirements but also perceptual requirements stemming from human senses, cognition, and physiology. Minimal and maximal perceptual requirements and limits must be factored into the engineering process (computing, processing, and so on). To do so, a new concept of quality-of-physical-experience (QoPE) measure is needed to merge physical factors from the human user itself with classical QoS (e.g., latency and rate) and QoE (e.g., mean-opinion score) inputs. Some factors that affect QoPE include brain cognition, body physiology, and gestures. As an example, in [2], we have shown that the human brain may not be able to distinguish between different latency measures, within the URLLC regime. Meanwhile, in [3], we showed that visual and haptic perceptions are key for maximizing resource utilization. Concisely, the requirements of $X R$ services are a blend of traditional URLLC and eMBB with incorporated perceptual factors that $6 \mathrm{G}$ must support.

Connected Robotics and Autonomous Systems (CRAS): A primary driver behind 6G systems is the imminent deployment of CRAS including drone-delivery systems, autonomous cars, autonomous drone swarms, vehicle platoons, and autonomous robotics. The introduction of CRAS over the cellular domain is not a simple case of "yet another short packet uplink loE service." Instead, CRAS mandate control system-driven latency requirements as well as the potential need for eMBB transmissions of high definition (HD) maps. The notion of QoPE applies once again for CRAS; however, the physical environment is now a con- 
XR will yield many killer applications for 6G across the AR/MR/VR spectrum. Upcoming 5G systems still

fall short of providing a full immersive XR experience capturing all sensory inputs due to their inability

to deliver very low latencies for data-rate intensive XR applications. A truly immersive AR/MR/VR

experience requires a joint design integrating not only engineering requirements but also perceptual

requirements stemming from human senses, cognition, and physiology

trol system, potentially augmented with AI. CRAS are perhaps a prime use case that requires stringent requirements across the rate-reliability-latency spectrum, a balance that is not yet available in $5 \mathrm{G}$.

Wireless Brain-Computer Interactions $(\mathrm{BCI})$ : Beyond $\mathrm{XR}$, tailoring wireless systems to their human users is mandatory to support services with direct $\mathrm{BCl}$. Traditionally, $\mathrm{BCl}$ applications were limited to healthcare scenarios in which humans can control prosthetic limbs or neighboring computing devices using brain implants. However, the recent advent of wireless brain-computer interfaces and implants will revolutionize this field and introduce new use-case scenarios that require 6G connectivity. Such scenarios range from enabling brain-controlled movie input to fully-fledged multibrain-controlled cinema [4]. Using wireless BCI technologies instead of smartphones, people will interact with their environment and other people using discrete devices, some worn, some implanted, and some embedded in the world around them. This will allow individuals to control their environments through gestures and communicate with loved ones through haptic messages. Such empathic and haptic communications, coupled with related ideas such as affective computing in which emotion-driven devices can match their functions to their user's mood, constitute important $6 \mathrm{G}$ use cases. Wireless $\mathrm{BCI}$ services require fundamentally different performance metrics compared to what 5G delivers. Similar to XR, wireless $\mathrm{BCl}$ services need high rates, ultra low latency, and high reliability. However, they are much more sensitive than XR to physical perceptions and necessitate QoPE guarantees.

Blockchain and Distributed Ledger Technologies (DLT): Blockchains and DLT will be one of the most disruptive IoE technologies. Blockchain and DLT applications can be viewed as the next-generation of distributed sensing services whose need for connectivity will require a synergistic mix of URLLC and massive machine type communications (mMTC) to guarantee low-latency, reliable connectivity, and scalability.

\section{G: Driving Trends and Performance Metrics}

The applications above lead to new system-wide trends that will set the goals for $6 \mathrm{G}$.

Trend 1 - More Bits, More spectrum, More Reliability: Most of the driving applications of $6 \mathrm{G}$ require higher bit rates than $5 \mathrm{G}$. To cater to applications such as $\mathrm{XR}$ and $\mathrm{BCl}$, 6G must deliver yet another 1000x increase in data rates yielding a target of around 1 Terabit/second. This motivates a need for more spectrum resources, hence prompting further exploration of frequencies beyond sub- $6 \mathrm{GHz}$. Meanwhile, the need for higher reliability will be pervasive across most $6 \mathrm{G}$ applications and will be more challenging to meet at high frequencies.
Trend 2 - From Areal to Volumetric Spectral and Energy Efficiency: 6G must deal with ground and aerial users, encompassing smartphones and $\mathrm{XR} / \mathrm{BCl}$ devices along with flying vehicles. This $3 \mathrm{D}$ nature of $6 \mathrm{G}$ requires an evolution toward a volumetric rather than spatial (areal) bandwidth definition. We envision that $6 \mathrm{G}$ systems must deliver high spectral and energy efficiency (SEE) requirements measured in bps $/ \mathrm{Hz} / \mathrm{m}^{3} /$ Joules. This is a natural evolution that started from $2 \mathrm{G}$ (bps) to $3 \mathrm{G}$ $(\mathrm{bps} / \mathrm{Hz})$, then $4 \mathrm{G}\left(\mathrm{bps} / \mathrm{Hz} / \mathrm{m}^{2}\right)$ to $5 \mathrm{G}(\mathrm{bps} / \mathrm{Hz} /$ $\mathrm{m}^{2}$ /Joules).

Trend 3 - Emergence of Smart Surfaces and Environments: Current and past cellular systems used base stations (of different sizes and forms) for transmission. We are witnessing a revolution in electromagnetically active surfaces (e.g., using metamaterials) that include man-made structures such as walls, roads, and even entire buildings, as exemplified by the Berkeley ewallpaper project (https://bwrc.eecs.berkeley.edu/projects/5605/ ewallpaper). The use of such smart large intelligent surfaces and environments for wireless communications will drive the $6 \mathrm{G}$ architectural evolution.

Trend 4 - Massive Availability of Small Data: The data revolution will continue in the near future and shift from centralized, big data, toward massive, distributed "small" data. 6G systems must harness both big and small datasets across their infrastructure to enhance network functions and provide new services. This trend motivates new machine learning techniques that go beyond classical big data analytics.

Trend 5 - From Self-Organizing Networks (SON) to Self-Sustaining Networks: SON has only been scarcely integrated into 4G/5G networks due to a lack of real-world need. However, CRAS and DLT technologies motivate an immediate need for intelligent SON to manage network operations, resources, and optimization. 6G will require a paradigm shift from classical SON, whereby the network merely adapts its functions to specific environment states, into a self-sustaining network (SSN) that can maintain its key performance indicators (KPIs), in perpetuity, under highly dynamic and complex environments stemming from the rich $6 \mathrm{G}$ application domains. SSNs must be able to not only adapt their functions but to also sustain their resource usage and management (e.g., by harvesting energy and exploiting spectrum) to autonomously maintain high, longterm KPIs. SSN functions must leverage the recent revolution in $\mathrm{Al}$ technologies to create Al-powered 6G SSNs.

Trend $6-$ Convergence of Communications, Computing, Control, Localization, and Sensing (3CLS): The past five generations of cellular systems had one exclusive function: wireless communications. However, 6G will disrupt this premise through a convergence (i.e., joint and simultaneous offering) of various functions that include communications, computing [5], control, localization, and sensing. We envision $6 \mathrm{G}$ as a multi-purpose system that can deliver multiple 3 CLS services that are particularly appealing and even necessary for applications such as XR, CRAS, and DLT where tracking, control, localization, and computing are an inherent feature. Moreover, sensing services will enable 6G systems to provide 
This article has been accepted for inclusion in a future issue of this magazine. Content is final as presented, with the exception of pagination.

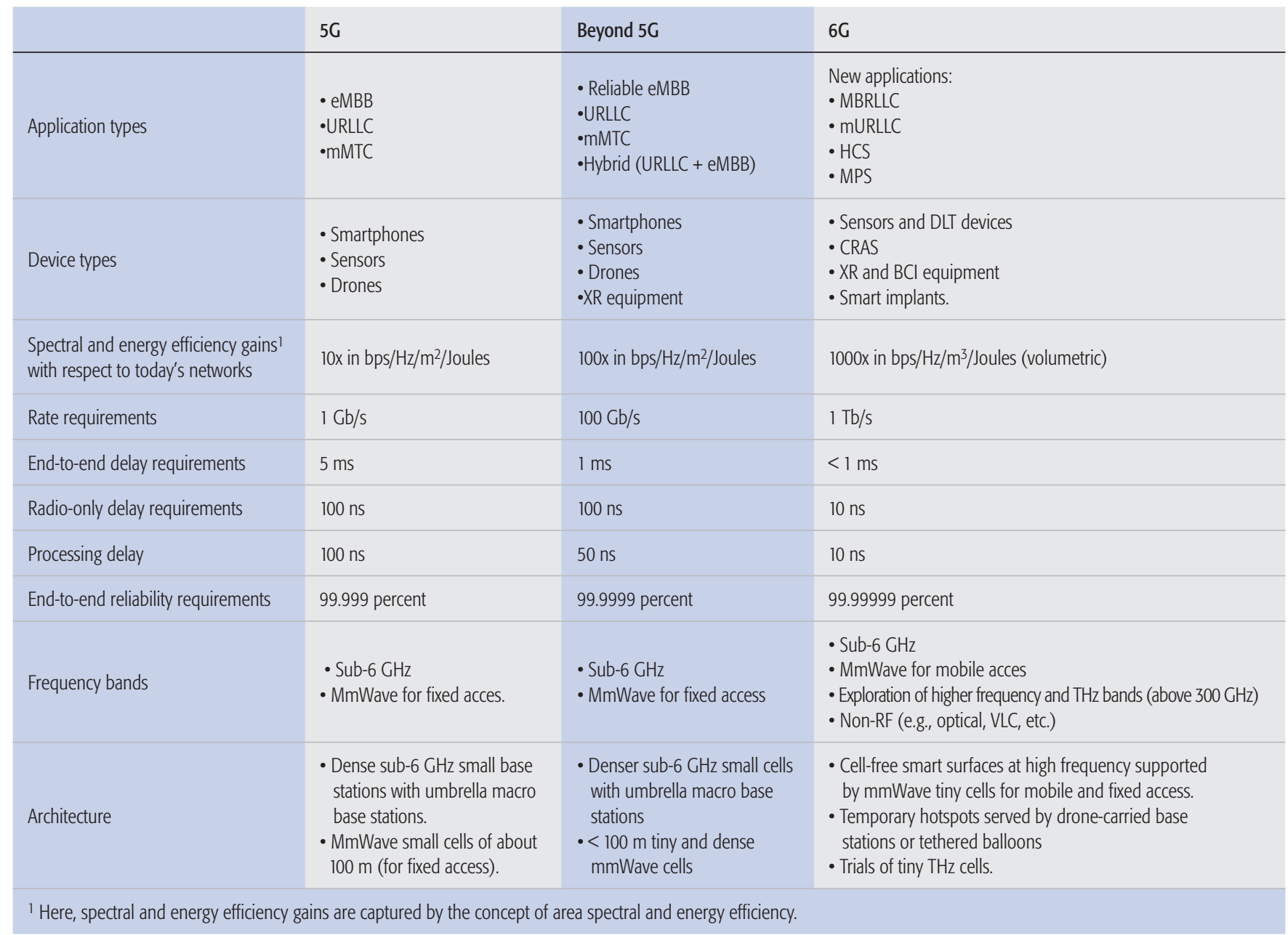

TABLE 1. Requirements of 5G vs. Beyond 5G vs. 6G.

users with a $3 D$ mapping of the radio environment across different frequencies. Hence, 6G systems must tightly integrate and manage 3CLS functions. Note that the evolutions pertaining to previous trends will gradually enable 6G systems to readily provide 3CLS.

Trend 7 - End of the Smartphone Era: Smartphones were central to $4 \mathrm{G}$ and 5G. However, in recent years there has been an increase in wearable devices whose functionalities are gradually replacing those of smartphones. This trend is further fueled by applications such as XR and $\mathrm{BCl}$. The devices associated with those applications range from smart wearables to integrated headsets and smart body implants that can take direct sensory inputs from human senses, bringing an end to smartphones and potentially driving a majority of 6G use cases.

As shown in Table 1, collectively, these trends impose new performance targets and requirements that will be met in two stages: a beyond $5 \mathrm{G}$ evolution, and a revolutionary 6G step.

\section{NeW GG SERVICE CLASSES}

Beyond imposing new performance metrics, the new technological trends will redefine 5G application types by morphing classical URLLC, eMBB, and $\mathrm{mMTC}$ and introducing new services (summarized in Table 2):

Mobile Broadband Reliable Low Latency Communication: As evident from above, the dis- tinction between eMBB and URLLC will no longer be sustainable to support applications such as $\mathrm{XR}$, wireless $\mathrm{BCl}$, or CRAS, because these applications require not only high reliability and low latency, but also high 5G-eMBB-level data rates. Hence, we propose a new service class called mobile broadband reliable low latency communication (MBRLLC) that allows 6G systems to deliver any required performance within the rate-reliability-latency space. As seen in Fig. 2, MBRLLC generalizes classical URLLC and eMBB services. Energy efficiency is central for MBRLLC, not only because of its impact on reliability and rate, but also because of the resource-limited nature of $6 \mathrm{G}$ devices.

Massive URLLC: 5G URLLC meant meeting reliability and latency of very specific uplink IoE applications such as smart factories, for which prior work [6] provided the needed fundamentals. However, 6G must scale classical URLLC across the device dimension, thereby leading to a new massive URLLC ( $m U R L L C)$ service that merges 5G URLLC with legacy mMTC. mURLLC brings forth a reliability-latency-scalability trade-off which mandates a major departure from average-based network designs (e.g., average throughput/delay). Instead, a principled and scalable framework that accounts for delay, reliability, packet size, architecture, topology (across access, edge, and core) and decision-making under uncertainty is necessary [7]. 
This article has been accepted for inclusion in a future issue of this magazine. Content is final as presented, with the exception of pagination.

\begin{tabular}{|c|c|c|}
\hline Service & Performance indicators & Example applications \\
\hline MBRLLC & $\begin{array}{l}\text { - Stringent rate-reliability-latency } \\
\text { requirements } \\
\text { - Energy efficiency } \\
\text { - Rate-reliability-latency in mobile } \\
\text { environments }\end{array}$ & $\begin{array}{l}\text {-XR/AR/VR } \\
\text { - Autonomous vehicular systems } \\
\text { - Autonomous drones } \\
\text { - Legacy eMBB and URLLC }\end{array}$ \\
\hline mURLLC & $\begin{array}{l}\text { - Ultra high reliability } \\
\text { - Massive connectivity } \\
\text { - Massive reliability } \\
\text { - Scalable URLLC }\end{array}$ & $\begin{array}{l}\text { - Classical Internet of Things } \\
\text { - User tracking } \\
\text { - Blockchain and DLT } \\
\text { - Massive sensing } \\
\text { - Autonomous robotics }\end{array}$ \\
\hline HCS & $\begin{array}{l}\text { - QoPE capturing raw wireless metrics as } \\
\text { well as human and physical factors }\end{array}$ & $\begin{array}{l}\text { - BC } \\
\text { - Haptics } \\
\text { - Empathic communication } \\
\text { - Affective communication }\end{array}$ \\
\hline MPS & $\begin{array}{l}\text { - Control stability } \\
\text { - Computing latency } \\
\text { - Localization accuracy } \\
\text { - Sensing and mapping accuracy } \\
\text { - Latency and reliability for communications } \\
\text { - Energy }\end{array}$ & $\begin{array}{l}\text { - CRAS } \\
\text { - Telemedicine } \\
\text { - Environmental mapping and imaging } \\
\text { - Some special cases of XR services }\end{array}$ \\
\hline
\end{tabular}

TABLE2. Summary of 6G service classes, their performance indicators, and example applications.

Human-Centric Services: We propose a new class of 6G services, dubbed human-centric services (HCS), that require QoPE targets (tightly coupled with their human users, as explained above) rather than raw rate-reliability-latency metrics. Wireless $\mathrm{BCl}$ are a prime example of HCS in which network performance is determined by the physiology of the human users and their actions. For such services, a whole new set of QoPE metrics must be defined and offered as a function of raw QoS and QoE metrics.

Multi-Purpose 3CLS and Energy Services: 6G systems must jointly deliver 3CLS services and their derivatives. They can also potentially offer energy to small devices via wireless energy transfer. Such multi-purpose 3CLS and energy services (MPS) will be particularly important for applications such as CRAS. MPS require joint uplink-downlink designs and must meet target performance for the control (e.g., stability), computing (e.g., computing latency), energy (e.g., target energy to transfer), localization (e.g., localization precision), and sensing and mapping functions (e.g., accuracy of a mapped radio environment).

\section{G: ENABLING TECHNOLOGIES}

To enable the aforementioned services and guarantee their performance, a cohort of new, disruptive technologies must be integrated into 6G.

Above $6 \mathrm{GHz}$ for $6 \mathrm{G}$ - from Small Cells to Tiny Cells: As per Trends 1 and 2, the need for higher data rates and SEE anywhere, anytime in 6G motivates exploring higher frequency bands beyond sub- $6 \mathrm{GHz}$. As a first step, this includes further developing mmWave technologies to make mobile mmWave a reality in early 6G systems. As 6G progresses, exploiting frequencies beyond mmWave, at the terahertz ( $\mathrm{THz}$ ) band, will become necessary [8]. To exploit higher mmWave and $\mathrm{THz}$ frequencies, the size of the $6 \mathrm{G}$ cells must shrink from small cells to "tiny cells"

whose radius is only a few tens of meters. This motivates new architectural designs that need much denser deployments of tiny cells and new high-frequency mobility management techniques.

Transceivers with Integrated Frequency Bands: On their own, dense high-frequency tiny cells may not be able to provide the seamless connectivity required for mobile $6 \mathrm{G}$ services. Instead, an integrated system that can leverage multiple frequencies across the microwave/mmWave/THz spectra (e.g., using multi-mode base stations) is needed to provide seamless connectivity at both wide and local area levels.

Communication with Large Intelligent Surfaces: Massive MIMO will be integral to both 5G and 6G due to the need for better SEE, higher data rates, and higher frequencies (Trend 1). However, for 6G systems, as per Trend 3, we envision an initial leap from traditional massive MIMO toward large intelligent surfaces (LISs) and smart environments [9] that can provide massive surfaces for wireless communications and for heterogeneous devices (Trend 7). LISs enable innovative ways for communication such as by using holographic radio frequency (RF) and holographic MIMO.

Edge Al: Al is witnessing an unprecedented interest from the wireless community [1] driven by recent breakthroughs in deep learning, the increase in available data (Trend 4), and the rise of smart devices (Trend 7). Imminent 6G use cases for Al (particularly for reinforcement learning) revolve around creating SSNs (Trend 5) that can autonomously sustain high KPIs and manage resources, functions, and network control. Al will also enable 6G to automatically provide MPS to its users and to send and create $3 \mathrm{D}$ radio environment maps (Trend 6). These short-term Al-enabled 6G functions will be complemented by a so called "collective network intelligence" in which network intelligence is pushed at the edge, running $\mathrm{Al}$ and learning algorithms on edge devices (Trend 7) to provide distributed autonomy. This new edge Al leap will create a $6 \mathrm{G}$ system that can integrate the services above, realize 3 CLS, and potentially replace classical frame structures.

Integrated Terrestrial, Airborne, and Satellite Networks: Beyond their inevitable role as $6 \mathrm{G}$ users, drones can be leveraged to complement terrestrial networks by providing connectivity to hotspots and to areas with scarce infrastructure. Meanwhile, both drones and terrestrial base stations may require satellite connectivity with low orbit satellites (LEO) and CubeSats to provide backhaul support and additional wide area coverage. Integrating terrestrial, airborne, and satellite networks [10] and [11] into a single wireless system will be essential for $6 \mathrm{G}$.

Energy Transfer and Harvesting: 6G could be the cellular system that can provide energy, along with 3CLS (Trend 6). As wireless energy transfer is maturing, we foresee $6 \mathrm{G}$ base stations providing basic power transfer for devices, particularly implants and sensors (Trend 7). Adjunct energy-centric ideas, such as energy harvesting and backscatter, will also be a component of $6 \mathrm{G}$.

Beyond 6G: A handful of technologies will mature along the same time of $6 \mathrm{G}$, and hence potentially play a role toward the end of the 6G standardization and research process. One prominent example is quantum computing and 
communications that can provide security and long-distance networking. Currently, major research efforts are focused on the quantum realm and we expect them to intersect with $6 \mathrm{G}$. Other similar beyond 6G technologies include integration of RF and non-RF links (including optical, neural, molecular, and other channels).

\section{GG: Research Agenda And Open Problems}

Building on the identified trends listed above and the enabling technologies, we now put forward a research agenda for 6G (Table 3).

3D Rate-Reliability-Latency Fundamentals: Fundamental 3D performance of $6 \mathrm{G}$ systems, in terms of rate-reliability-latency trade-offs and SEE, is needed. Such analysis must quantify the spectrum, energy, and communication requirements that 6G needs in order to support the identified driving applications. Recent works in [7] and [12] provide a first step in this direction.

Exploring Integrated, Heterogeneous High-Frequency Bands: Exploiting mmWave and $\mathrm{THz}$ in 6G brings forth several new open problems. For mmWave, supporting high mobility at mmWave frequencies will be a central open problem. For $\mathrm{THz}$, new transceiver architectures and propagation models are needed [8]. High power, high sensitivity, and low noise figure are key transceiver features needed to overcome the very high $\mathrm{THz}$ path loss. Once these physical layer aspects are well-understood, there is a need to develop new network and link-layer protocols to optimize the use of cross-frequency resources while taking into account the highly varying and uncertain nature of the mmWave and $\mathrm{THz}$ environments. Another important direction is to study the co-existence of $\mathrm{THz}, \mathrm{mmW}$ ave, and microwave cells across all layers, building on early works such as [13].

3D Networking: Due to the integration of ground and airborne networks, as outlined above, 6G must support communications in 3D space, including serving users in 3D and deploying 3D base stations (e.g., tethered balloons or temporary drones). This requires concerted research on various fronts. First, measurement and (data-driven) modeling of the $3 \mathrm{D}$ propagation environment is needed. Second, new approaches for 3D frequency and network planning (e.g., where to deploy base stations, tethered balloons, or even dronebase stations) must be developed. Our work in [10] showed that such 3D planning is substantially different from conventional 2D networks due to the new altitude dimension and the associated degrees of freedom. Finally, new network optimizations for mobility management, routing, and resource management in 3D are needed.

Communication with LIS: As per Trend 3, 6G will provide wireless connectivity via smart LIS environments that include active frequency selective surfaces, metallic passive reflectors, passive/ active reflect arrays, as well as nonreconfigurable and reconfigurable metasurfaces. Open problems here range from the optimized deployment of passive reflectors and metasurfaces to Al-powered operation of LISs. Fundamental analysis to understand the performance of smart surfaces, in terms of rate, latency, reliability, and coverage is needed, building on the early work in [9]. Another important direction is to investigate the

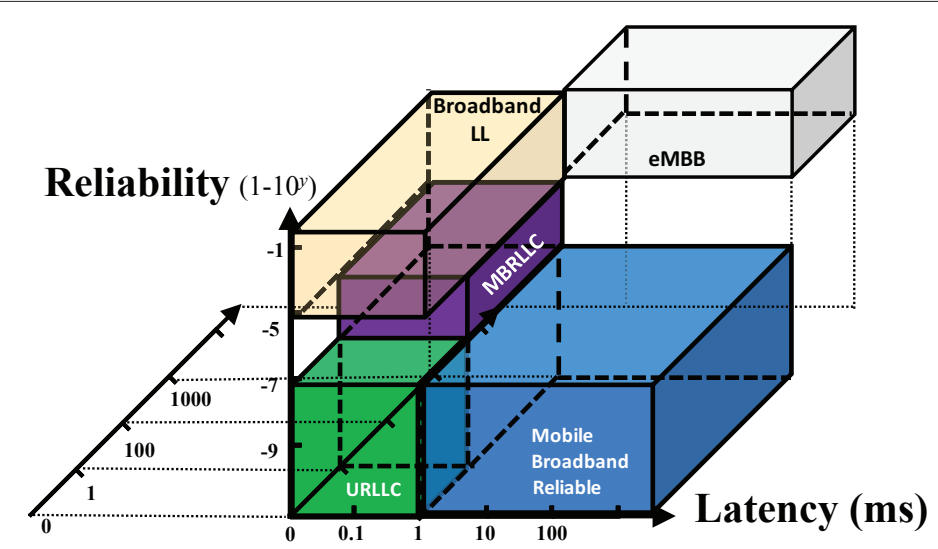

\section{Data rate (Gbps)}

FIGURE 2. MBRLLC services and several special cases (including classical eMBB and URLLC) within the rate-reliability-latency space. Other involved, associated metrics that are not shown include energy and network scale.

potential of using LIS-based reflective surfaces to enhance the range and coverage of tiny cells and to dynamically modify the propagation environment.

Al for Wireless: Al brings forward many major research directions for $6 \mathrm{G}$. Beyond the need for massive, small data analytics as well as using machine learning (ML) and Al-based SSNs (realized using reinforcement learning and game theory), there is also a need to operate $\mathrm{ML}$ algorithms reliably over $6 \mathrm{G}$ to deliver the applications listed previously. To perform these critical application tasks, low-latency, high-reliability and scalable AI is needed, along with a reliable infrastructure [1] and [3]. This joint design of ML and wireless networks is a key $6 \mathrm{G}$ research area.

QoPE Metrics: The design of QoPE metrics that integrate physical factors from human physiology (for HCS services) or from a control system (for CRAS) is an important 6G research area, especially in light of new, emerging devices (Trend 7). This requires both real-world psychophysics experiments as well as new, rigorous mathematical expressions for QoPE that combine QoS, QoE, and human perceptions. Theoretical development of QoPE can be achieved using techniques from other disciplines such as operations research (e.g., multi-attribute utility theory (see [14])) and machine learning (see [2]). 6G will be the first generation to enable a new breed of applications (wireless $\mathrm{BCl}$ ) leveraging multiple human cognitive senses.

Joint Communication and Control: $6 \mathrm{G}$ needs to pervasively support CRAS. The performance of CRAS is governed by real-world control systems whose operation requires data input from wireless 6G links. Therefore, operating CRAS over 6G systems requires a communication and control co-design, whereby the performance of the $6 \mathrm{G}$ wireless links is optimized to cater to the stability of the control system and vice versa. Due to the traditional radio-centric focus (3GPP and IEEE fora), such a co-design has been overlooked in 5G. Meanwhile, prior works on networked control abstract the specifics of the wireless network and cannot apply to cellular communications. This makes the communication-control co-design a key research topic in $6 \mathrm{G}$. 
This article has been accepted for inclusion in a future issue of this magazine. Content is final as presented, with the exception of pagination.

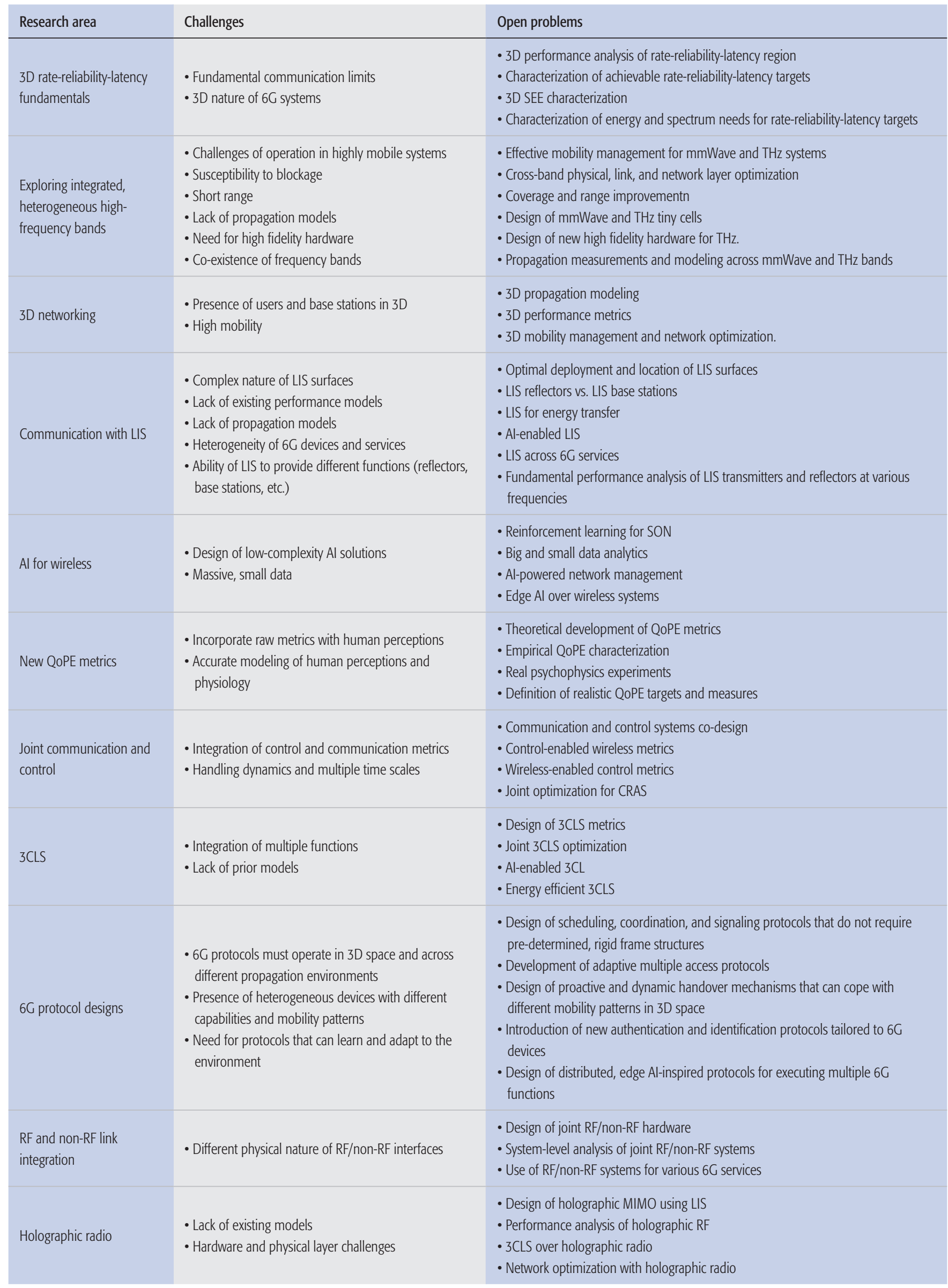

TABLE 3. Summary of research areas. 
3CLS: The idea of joint communication and control must be extended to the joint design of the entire 3CLS functions. The interdependence between computing, communication, control, localization, sensing, energy, and mapping has not yet been fully explored in an end-to-end manner. Key questions range from how to jointly meet the performance of all 3CLS services to multi-modal sensor fusion for reconstructing 3D images and navigating in unknown environments for navigating robots, autonomous driving, and so on. 3CLS is needed for various applications including CRAS, $\mathrm{XR}$, and DLT.

6G Protocol Designs: Owing to all trends discussed previously and their challenges, compared to 5G, 6G will require radical new protocol designs. For instance, 6G must introduce new, Al-driven protocols for signaling, scheduling, and coordination that can replace conventional 5G protocols that rely on pre-determined network parameters and rigid frame structures. These new 6G protocols will, in contrast, continuously adapt to the current and projected state of the wireless environment. As 6G evolves, there will be a need for new, dynamic multiple access [15] protocols that can dynamically change the type of multiple access (orthogonal or non-orthogonal, random or scheduled) used depending on the needs of the applications and the network state. Moreover, novel handover protocols must be designed to account for the 3D nature of the $6 \mathrm{G}$ system and the presence of different types of mobile devices. New protocols for authentication and identification will also be needed to handle the new breed of wireless devices that include drones, vehicles, as well as embedded and implanted devices. Finally, all 6G protocols must be distributed and able to leverage datasets distributed across the network edge.

RF and Non-RF Link Integration: 6G will witness a convergence of RF and non-RF links that encompass optical, visible light communication (VLC), molecular communication, and neuro-communication, among others. Design of such joint $\mathrm{RF} /$ non-RF systems is an open research area.

Holographic Radio: RF holography (including holographic MIMO) and spatial spectral holography can be made possible with $6 \mathrm{G}$ due to the use of LIS and similar structures. Holographic RF allows for control of the entire physical space and the full closed loop of the electromagnetic field through spatial spectral holography and spatial wave field synthesis. This greatly improves spectrum efficiency and network capacity, and helps the integration of imaging and wireless communication. How to realize holographic radio is a widely open area.

An overview on the necessary analytical tools and fundamentals related to these open problems is shown in Fig. 3.

\section{Conclusion and ReCommendations}

This article laid out a bold new vision for $6 \mathrm{G}$ systems that outlines the trends, challenges, and associated research. While many topics will come as a natural 5G evolution, new avenues of research such as LIS communication, 3CLS, holographic radio, and others will create an exciting research agenda for the next decade. We conclude with several recommendations:

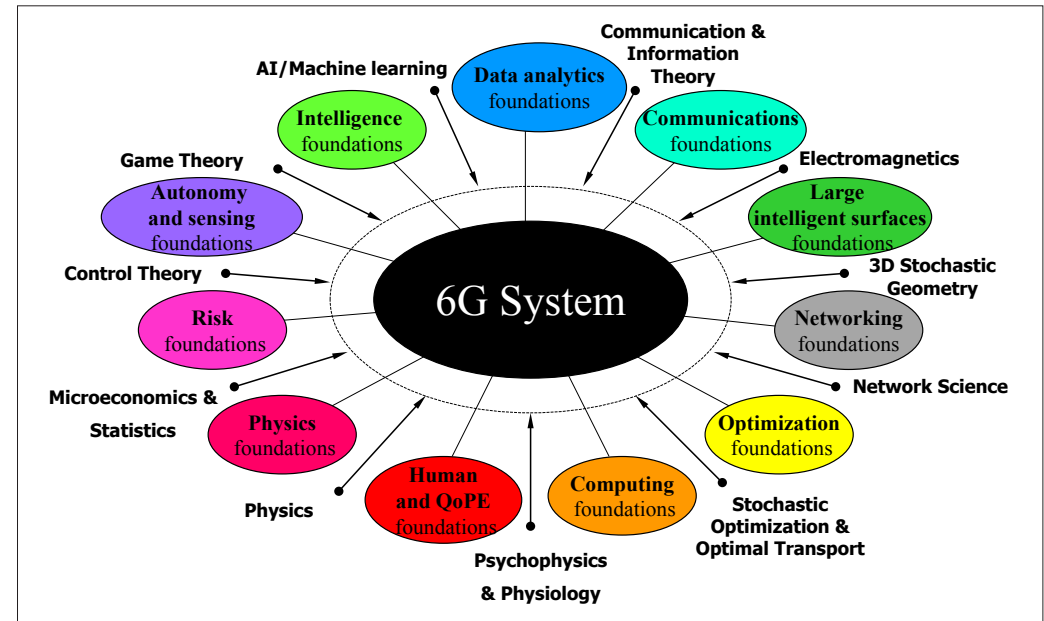

FIGURE 3. Necessary foundations and associated analytical tools for 6G.

- Recommendation 1: A first step toward 6G is to enable MBRLLC and mobility management at high-frequency mmWave bands and beyond (i.e., THz).

- Recommendation 2: 6G requires a move from radio-centric system design (i.e., 3GPP) toward an end-to-end 3CLS co-design under the orchestration of an Al-driven intelligence substrate.

- Recommendation 3: The 6G vision will not be a simple case of exploring additional, high-frequency spectrum bands to provide more capacity. Instead, it will be driven by a diverse portfolio of applications, technologies, and techniques (Figs. 1 and 3).

- Recommendation 4: 6G will transition from the smartphone-base station paradigm into a new era of smart surfaces communicating with human-embedded implants.

- Recommendation 5: Performance analysis and optimization of $6 \mathrm{G}$ requires operating in 3D space and moving away from simple averaging toward fine-grained analysis that deals with tails, distributions, and QoPE.

\section{ACKNOWLEDGMENT}

This research was supported by the U.S. National Science Foundation under Grant CNS-1836802. It was also supported in part by the Academy of Finland project CARMA; the Academy of Finland project MISSION; the Academy of Finland project SMARTER; the INFOTECH project NOOR; the Nokia Bell-Labs project FOGGY; and the Nokia Foundation.

\section{REFERENCES}

[1] M. Chen et al., "Artificial Neural Networks-Based Machine Learning for Wireless Networks: A Tutorial," IEEE Commun. Surveys \& Tutorials, to appear, 2019.

[2] A. Taleb Zadeh Kasgari, W. Saad, and M. Debbah, "Humanin-the-Loop Wireless Communications: Machine Learning and Brain-Aware Resource Management," IEEE Trans. Commun., to appear, 2019.

[3] J. Park et al., "Wireless Network Intelligence at the Edge," arXiv preprint arXiv:1812.02858, Dec. 2018.

[4] P. Zioga et al., "Enheduanna a Manifesto of Falling Live Brain Computer Cinema Performance: Performer and Audience Participation, Cognition and Emotional Engagement Using Multi Brain BCl Interaction," Frontiers in Neuroscience, vol. 12, April 2018, pp. 191.

[5] Y. Li et al., "Joint Optimization of Radio and Virtual Machine Resources with Uncertain User Demands in Mobile Cloud Computing," IEEE Trans. Multimedia, vol. 20, no. 9, Sept. 2018, pp. 2427-38. 
[6] G. Durisi, T. Koch, and P. Popovski, "Toward Massive, Ultrareliable, and Low-Latency Wireless Communication with Short Packets," Proc. IEEE, vol. 104, no. 9, Sept. 2016, pp. 1711-26.

[7] M. Bennis, M. Debbah, and H. V. Poor, "Ultrareliable and Low-Latency Wireless Communication: Tail, Risk, and Scale," Proc. IEEE, vol. 106, no. 10, Oct. 2018, pp. 1834-53.

[8] Y. Xing and T. S. Rappaport, "Propagation Measurement System and Approach at $140 \mathrm{GHz}$-Moving to 6G and Above $100 \mathrm{GHz}$," Proc. IEEE Global Commun. Conf. (GLOBECOM), Abu Dhabi, United Arab Emirates, Dec. 2018.

[9] S. Hu, F. Rusek, and O. Edfors, "Beyond Massive MIMO: The Potential of Data Transmission with Large Intelligent Surfaces," IEEE Trans. Signal Processing, vol. 66, no. 10, May 2018, pp. 2746-58.

[10] M. Mozaffari et al., "Beyond 5G with UAVs: Foundations of a 3D Wireless Cellular Network," IEEE Trans. Wireless Commun vol. 18, no. 1 , Jan. 2019, pp. 357-72

[11] X. Cao et al., "Guest Editorial Airborne Communication Networks," IEEE J. Selected Areas in Commun., vol. 36, no. 9, Sept. 2018, pp. 1903-06.

[12] A. T. Z. Kasgari and W. Saad, "Model-Free Ultra Reliable Low Latency Communication (URLLC): A Deep Reinforcement Learning Framework," Proc. IEEE Inr'l. Conf. Commun. (ICC), Shanghai, China, May 2019

[13] O. Semiari et al., "Integrated Millimeter Wave and Sub-6 $\mathrm{GHz}$ Wireless Networks: A Roadmap for Joint Mobile Broadband and Ultra-Reliable Low-Latency Communications," IEEE Wireless Commun., vol. 26, no. 2, Apr. 2019, pp. 109-15.

[14] M. Chen, W. Saad, and C. Yin, "Virtual Reality over Wireless Networks: Quality-of-Service Model and Learning-Based Resource Management," IEEE Trans. Commun., vol. 66, no. 11, Nov. 2018, pp. 5621-35.

[15] B. Cao et al. "Intelligent Offloading in Multi-Access Edge Computing: A State-of-the-Art Review and Framework," IEEE Commun. Mag., vol. 57, no. 3, Mar. 2019, pp. 56-62.

\section{BIOGRAPHIES}

WALID SAAD [S'07, M'10, SM'15, F'19] received his Ph.D degree from the University of Oslo in 2010. Currently, he is a professor with the Department of Electrical and Computer Engineering at Virginia Tech, where he leads the Network sciEnce, Wireless, and Security (NEWS) laboratory. His research interests include wireless networks, machine learning, game theory, cybersecurity, unmanned aerial vehicles, cellular networks, and cyber-physical systems. He is the author/co-author of eight conference best paper awards and of the 2015 IEEE ComSoc Fred W. Ellersick Prize. He is an IEEE Fellow and an IEEE Distinguished Lecturer.

MEHDI BENNIS [SM'15] is an associate professor at the Centre for Wireless Communications, University of Oulu, Finland, and an Academy of Finland Research Fellow. He has been the recipient of several awards, including the Fred W. Ellersick Prize and Best Tutorial Prize from the IEEE Communications Society and EURASIP Best Paper Award for the Journal of Wireless Communications and Networks. He is currently an editor of IEEE Transactions on Communications.

MINGZHE CHEN received the Ph.D. degree from the Beijing University of Posts and Telecommunications, Beijing, China, in 2019. From 2016 to 2019, he was a visiting researcher at the Department of Electrical and Computer Engineering, Virginia Tech. He is currently a postdoctoral fellow at the Chinese University of Hong Kong, Shenzhen, China, and at the Electrical Engineering Department of Princeton University. His research interests include machine learning, virtual reality, unmanned aerial vehicles, game theory, wireless networks, and caching. He was an exemplary reviewer for IEEE Transactions on Communications in 2018. 